\title{
Unfavorable Outcome of Percutaneous Coronary Intervention for Coronary Heart Disease with Rare Severe Pericoronary Fat Stranding
}

\author{
Hui Hui ${ }^{1}$, Yousheng Yuan ${ }^{2}$, Zhaoqian Wang2, Yana Dou ${ }^{3}$, Xixia Sun ${ }^{2}$, \\ Yujie Zou ${ }^{2}$, Chongfu Jia ${ }^{2 *}$ \\ ${ }^{1}$ Department of Cardiology, Dalian Municipal Central Hospital Affiliated of Dalian Medical University, Dalian, China \\ ${ }^{2}$ Department of Cardiovascular Radiology, The First Affiliated Hospital of Dalian Medical University, Dalian, China \\ ${ }^{3}$ Diagnostic Imaging, Siemens Healthcare Ltd., Beijing, China \\ Email: *chongfujia@sina.com
}

How to cite this paper: Hui, H., Yuan, Y.S., Wang, Z.Q., Dou, Y.N., Sun, X.X., Zou, Y.J. and Jia, C.F. (2021) Unfavorable Outcome of Percutaneous Coronary Intervention for Coronary Heart Disease with Rare Severe Pericoronary Fat Stranding. World Journal of Cardiovascular Diseases, 11, 292-297.

https://doi.org/10.4236/wjcd.2021.116028

Received: May 24, 2021

Accepted: June 19, 2021

Published: June 22, 2021

Copyright $\odot 2021$ by author(s) and Scientific Research Publishing Inc. This work is licensed under the Creative Commons Attribution International License (CC BY 4.0).

http://creativecommons.org/licenses/by/4.0/

\begin{abstract}
Background: Pericoronary fat stranding (PCFS) is a novel noninvasive imaging sign of coronary inflammation, and has important implications for cardiovascular risk stratification, and the outcome of percutaneous coronary intervention (PCI) for coronary heart disease patients with pericoronary fat stranding remains unknown. Aim: We report two rare cases of PCI-treated coronary heart disease patients with severe PCFS. Case Presentation: This is the first report of two rare cases of PCI for patients with coronary heart disease surrounded by severe PCFS. We demonstrated that the outcome in these cases is poor, and in-stent restenosis or occlusion occurs rapidly within $2-6$ months following PCI. However, Case 2 underwent coronary artery bypass grafting after the rapid occurrence of in-stent occlusion, and the bypass graft remained intact over the 5 years following coronary artery bypass grafting. Conclusion: Coronary artery bypass grafting rather than PCI may be appropriate for coronary heart disease patients with severe PCFS.
\end{abstract}

\section{Keywords}

Computed Tomographic Angiography, Coronary Artery Disease, Pericoronary Fat, Percutaneous Coronary Intervention, Outcome

\section{Introduction}

Pericoronary fat stranding (PCFS) is defined as an irregular obscuration caused 
by an abnormally increased CT attenuation of pericoronary fat, occurring as a manifestation of edema, inflammation or neoplastic infiltration [1]. Recent studies have shown that PCFS is closely associated with coronary inflammation, vulnerable plaques, plaque progression and cardiovascular events, and PCFS can be a potential imaging biomarker to monitor the anti-inflammation response to statin treatments [1] [2] [3]. However, the outcome of percutaneous coronary intervention (PCI) for coronary heart disease patients with PCFS remains unknown. We report two rare cases of PCI-treated coronary heart disease patients with severe PCFS.

\section{Case Report and Case Presentation}

\subsection{Case 1}

A 63-year-old female initially presented to our hospital with repeated retrosternal crushing pain. She reported a 5-year history of hypertension which was well controlled; she denied any history of diabetes, smoking, drinking of alcohol, allergies and familial genetic diseases. On admission, her ECG showed abnormal $\mathrm{Q}$ waves in leads III and avF (Figure 1). The laboratory examination showed: an elevated hypersensitive cardiac troponin I of $1.80 \mu \mathrm{g} / \mathrm{L}$, total cholesterol of 5.66 $\mathrm{mmol} / \mathrm{L}$, low density lipoprotein of $3.54 \mathrm{mmol} / \mathrm{L}$, hypersensitive C-reactive protein of $4.9 \mathrm{mg} / \mathrm{L}$, and erythrocyte sedimentation rate of $32 \mathrm{~mm} / \mathrm{h}$. Coronary computed tomography angiography (CCTA) demonstrated occlusion in the proximal left anterior descending (LAD) artery with severe PCFS, moderate stenosis in the proximal left circumflex (LCX) artery with PCFS, and no lesion in the right coronary artery (RCA) (Figures $2(\mathrm{a})-(\mathrm{d})$ ). The LAD was then treated with a drug-eluting stent, and her symptoms resolved. However, 2 months later, the patient was admitted to our hospital with a recurrence of retrosternal paina

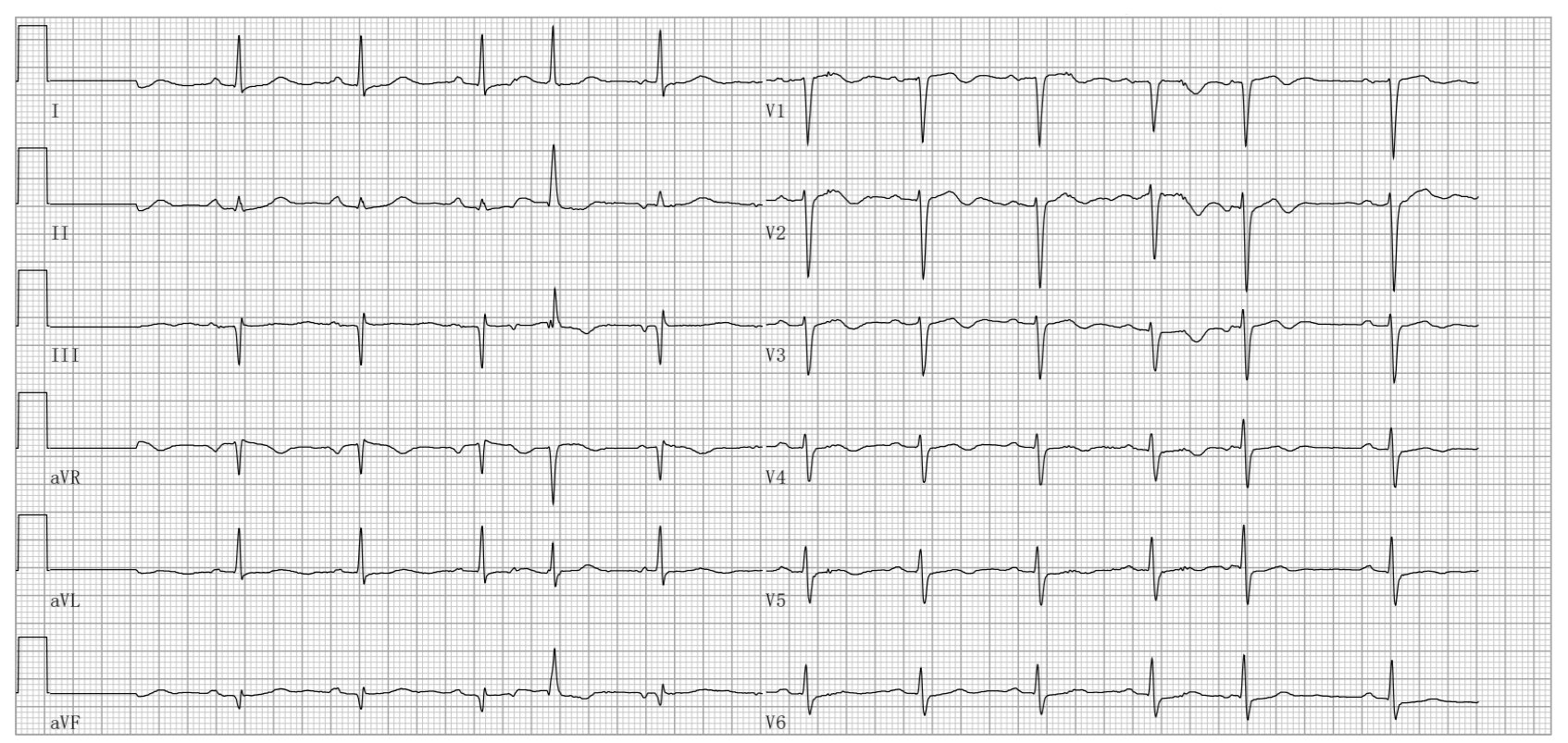

Figure 1. The ECG images from Case 1. The ECG showed abnormal Q waves in leads III and avF. 


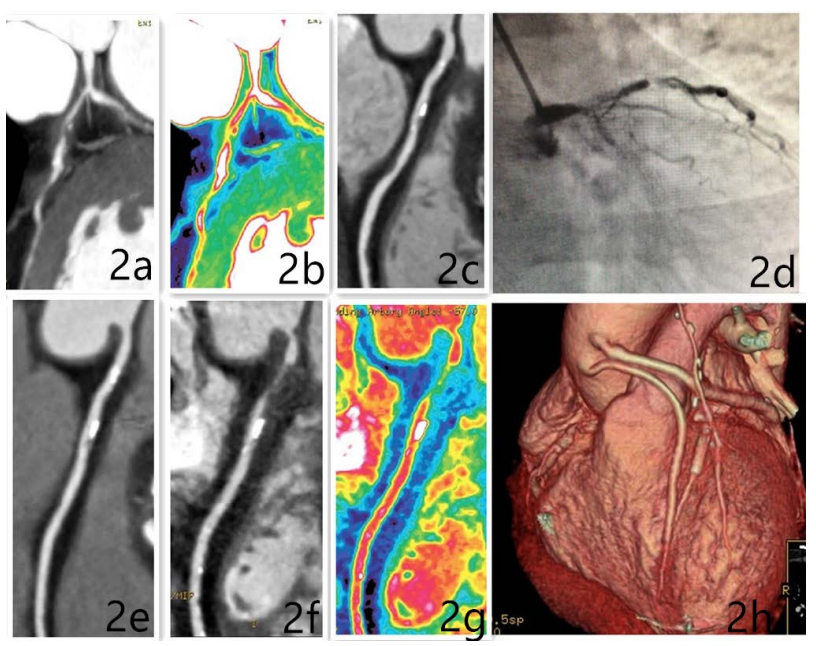

Figure 2. Serial CCTA and CAG images from Case 1. The baseline CCTA (2017-6) in (a) (CPR), (b) (corresponding colorized image), (c) (CPR) and (d) (CPR) indicated severe PCFS with occlusion in LAD, PCFS with moderate stenosis in LCX and no lesion in RCA separately (arrow). The first follow-up CCTA (2017-8) in (e) (CPR) indicated PCFS increase with stenosis progression to a severe degree in LCX (arrow). The intravascular ultrasound (2017-8) in (f) indicated good stent placement in LCX. The follow-up CAG (2017-12) in (g) indicated in-stent occlusion in LAD and severe restenosis in LCX (arrow). The second follow-up CCTA (2018-8) in (h) (CPR) and (i) (corresponding colorized image) indicated new severe PCFS and stenosis in RCA (arrow). CCTA, coronary computed tomographic angiography; CAG, catheterization angiography; CPR, curved planar reconstruction; LAD, left anterior descending artery; LCX, left circumflex artery; PCFS, perivascular fat stranding; RCA, right coronary artery.

gain. CCTA indicated LAD stent patency and normal RCA, and in the LCX, rapidly increased PCFS with progression of stenosis to subocclusion (Figure $2(e))$. Subsequent intravascular ultrasound showed fatty dominant plaques in the LCX. The LCX was also then treated with a drug-eluting stent, and intravascular ultrasound indicated good stent placement (Figure 2(f)). Unfortunately, 4 months later, catheterization angiography showed that in the LAD there had been rapid progression to in-stent occlusion as well as severe restenosis in the LCX (Figure 2(g)), On several occasions, coronary artery bypass grafting was suggested to the patient, but she refused, and following this, the patient was admitted to hospital 9 times, once every 2 - 6 months, due to repeated and rapid in-stent restenosis or occlusion. Moreover, CCTA during her 4th hospital admission (14 months after the initial hospital admission) showed new and severe PCFS with severe stenosis in the proximal RCA (Figures 2(h)-(i)). The patient underwent re-implantation stent, balloon angioplasty including drug-coated balloon, and reinforcement drug treatment including PCSK9, but the outcomes 
were not good.

\subsection{Case 2}

A 55-year-old female initially presented to our hospital with intermittent precordial crushing pain. She reported a 4-year history of hypertension which was well controlled; she denied any history of diabetes, hyperlipidemia, smoking, drinking alcohol, allergies and familial genetic diseases. On admission, ECG indicated ST segment depression and giant T wave inversion (Figure 3). The laboratory examination showed elevated creatine kinase of $220 \mathrm{IU} / \mathrm{L}$, hypersensitive cardiac troponin I of $3.095 \mu \mathrm{g} / \mathrm{L}$, CK-MB of $17.7 \mathrm{mg} / \mathrm{L}$, hypersensitive C-reactive protein of $17.7 \mathrm{mg} / \mathrm{L}$, and erythrocyte sedimentation rate of $27 \mathrm{~mm} / \mathrm{h}$. CCTA showed occlusion in the proximal LAD and LCX with severe PCFS, moderate stenosis in left main coronary artery (LMA) with PCFS, and mild stenosis in the mid RCA (Figures 4(a)-(c)). The LMA, LAD and LCX arteries were treated with drug-eluting stents, and her symptoms resolved. However, 3 months later, she was admitted to our hospital with precordial painagain, and subsequent catheterization angiography showed rapid progression to in-stent occlusion in the LMA, LAD and LCX (Figure 4(d)). Therefore, coronary artery bypass grafting was then performed for the LAD, diagonal branch and LCX. One year later, follow-up CCTA indicated all three grafts were intact, and there was new mild PCFS and stenosis in the proximal RCA (Figure 4(e)). Four years later, both CCTA and catheterization angiography during her 3rd hospital admission indicated all three grafts were still intact, while the PCFS in the RCA had significantly increased with the severe progression of the stenosis. The RCA was then treated with a drug-eluting stent (Figures $4(\mathrm{f})-(\mathrm{h})$ ).

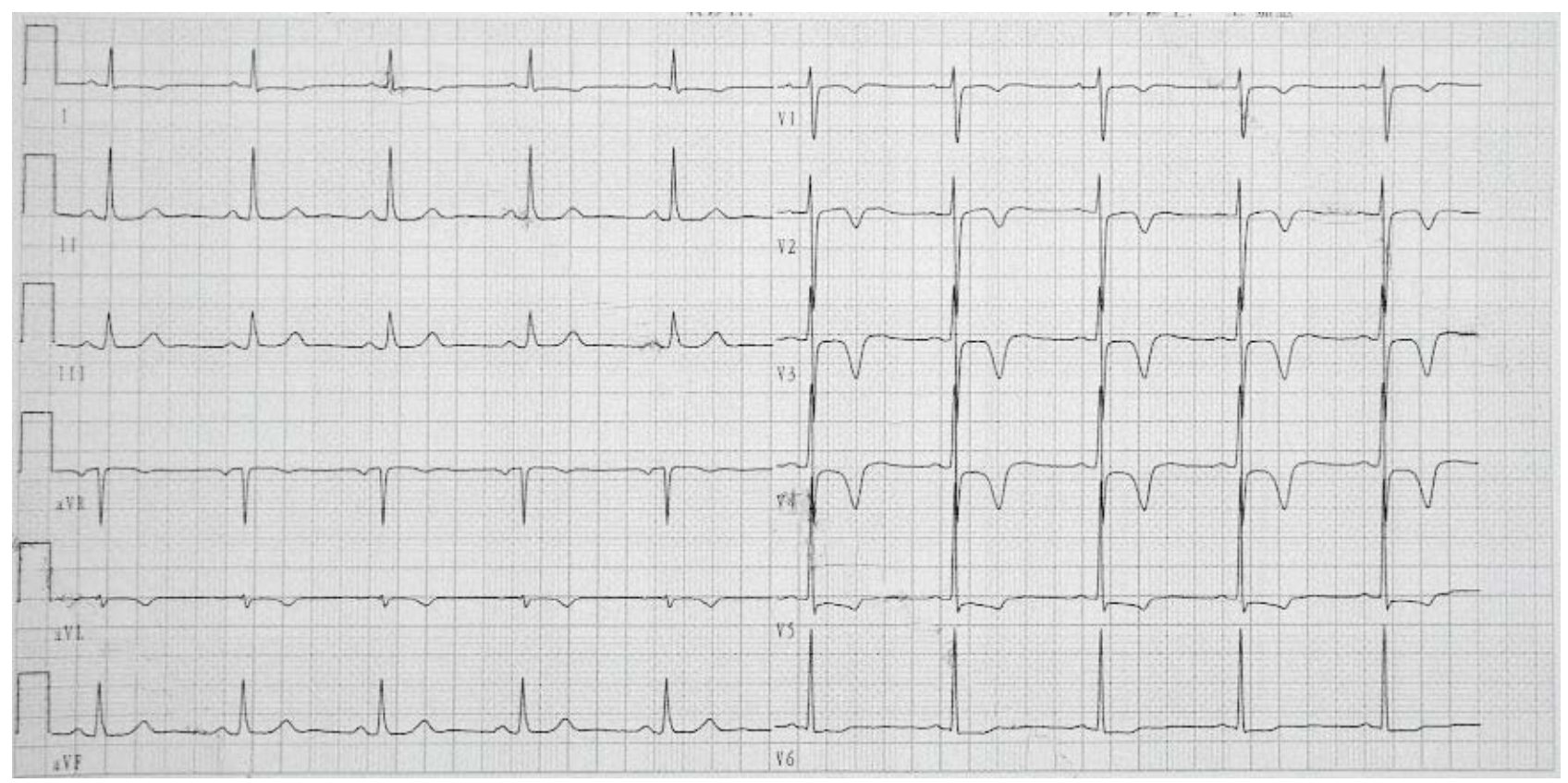

Figure 3. The ECG images from Case 2. The ECG showed ST segment depression and giant T wave inversion. 


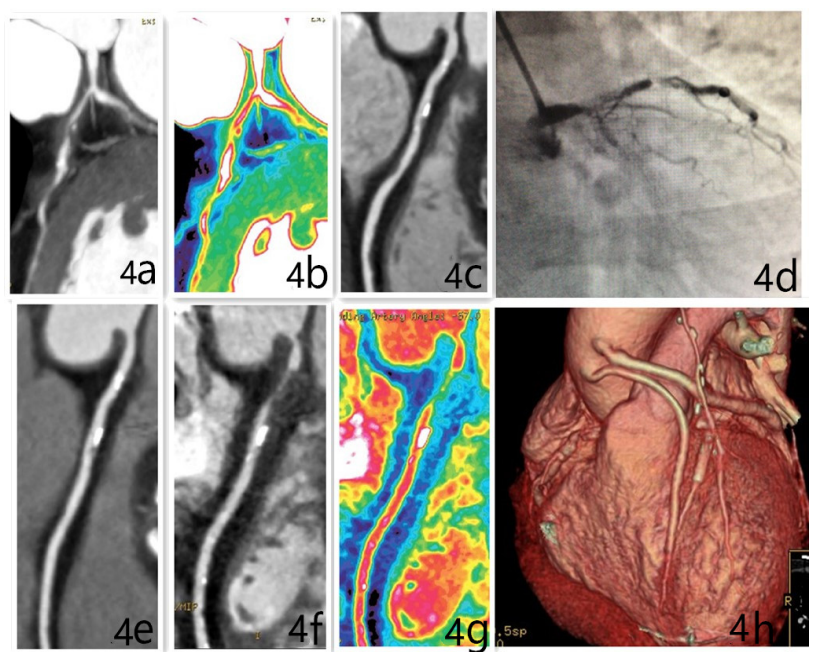

Figure 4. Serial CCTA and CAG images from Case 2. The baseline CCTA (2014-8) in (a) (CPR) and (b) (corresponding colorized image) indicated severe PCFS in LMA, LAD and LCX (arrow). The baseline CCTA in (c) (CPR) indicated mild stenosis in mid RCA without PCFS (arrow). Follow-up CAG (2014-11) in (d) indicated in-stent occlusion in LMA, LAD and LCX (arrow). The first follow-up CCTA (2016-1) in (e) (CPR) indicated new mild PCFS and stenosis in RCA (arrow). The second follow-up CCTA (2019-10) in (f) (CPR), (g) (corresponding colorized image) and (h) (volume reconstruction) indicated PCFS increased significantly with severe stenosis in RCA and three grafts to the LAD, diagonal and LCX were intact 5 years after coronary artery bypass grafting separately (arrow). LMA, left main coronary artery. Other abbreviations are as in Figure 2.

\section{Discussion}

This is the first report of cases of PCI for rare patients with coronary heart disease surrounded by severe PCFS. We demonstrated that the outcome in these cases maybe poor, and in-stent restenosis or occlusion occurs rapidly within 2 6 months following PCI.

In Case 1, multiple therapies were tried following PCI, but they failed to arrest the rapid development of in-stent restenosis. The mechanism at work may be that proinflammatory adipokines and cytokines were secreted as a byproduct of the PCFS, and these directly diffused into the vascular walls due to the lack of a fascial plane, and this induced atherosclerosis-vascular inflammation, further causing in-stent atherosclerosis and restenosis [4]. Case 2 differs from Case 1 in that Case 2 underwent coronary artery bypass grafting after the rapid occurrence of in-stent occlusion, and the bypass graft remained intact over the 5 years following coronary artery bypass grafting. Another interesting finding is that in 2 Cases, severe PCFS was observed initially in the LAD and LCX arteries, and then PCFS in the RCA occurred about 1 year later. Furthermore, PCFS increased over time, accompanied by the rapid progression of stenosis. 


\section{Conclusion}

We suggested that the outcome of PCI-treated coronary heart disease patients with uncommon severe PCFS is poor, and coronary artery bypass grafting may be appropriate for these patients. Furthermore, severe PCFS is ultimately prone to involve all three coronary arteries. Further study should be required to confirm and refine our findings.

\section{Acknowledgements}

The authors have no acknowledgement to declare.

\section{Statement of Ethics}

Written informed consent was obtained from the patient to publish their case (including publication of images).

\section{Funding Sources}

No funding was received for this work.

\section{Conflicts of Interest}

The authors have no conflicts of interest to declare.

\section{References}

[1] Hedgire, S., Baliyan, V., Zucker, E.J., Bittner, D.O., Staziaki, P.V., Takx, R.A.P., et al. (2018) Perivascular Epicardial Fat Stranding at Coronary CT Angiography: A Marker of Acute Plaque Rupture and Spontaneous Coronary Artery Dissection. Radiology, 287, 808-815. https://doi.org/10.1148/radiol.2017171568

[2] Kwiecinski, J., Dey, D., Cadet, S., Lee, S.E., Otaki, Y., Huynh, P.T., et al. (2019) Peri-Coronary Adipose Tissue Density Is Associated with ${ }^{18} \mathrm{~F}$-Sodiumfluoride Coronary Uptake in Stable Patients with High-Risk Plaques. JACC: Cardiovascular Imaging, 12, 2000-2010. https://doi.org/10.1016/j.jcmg.2018.11.032

[3] Oikonomou, E.K., Marwan, M., Desai, M.Y., Mancio, J., Alashi, A., Hutt Centeno, E., et al. (2018) Non-Invasive Detection of Coronary Inflammation Using Computed Tomography and Prediction of Residual Cardiovascular Risk (the CRISP CT Study): A Post-Hoc Analysis of Prospective Outcome Data. Lancet, 392, 929-939. https://doi.org/10.1016/S0140-6736(18)31114-0

[4] Britton, K.A. and Fox, C.S. (2011) Perivascular Adipose Tissue and Vascular Disease. Journal of Clinical Lipidology, 6, 79-91. https://doi.org/10.2217/clp.10.89 